\title{
Clave para los géneros de Boraginaceae sensu lato nativos del Brasil
}

\author{
José Iranildo Miranda de Melo ${ }^{1 *}$ \\ María Natividad Sánchez de Stapf ${ }^{2}$ \\ ${ }^{1}$ Universidade Estadual da Paraíba, Centro de Ciências Biológicas e da Saúde Departamento de Biologia \\ CEP 58429-500, Campina Grande - PB, Brasil \\ ${ }^{2}$ Universidad de Panamá, Facultad de Ciencias Naturales \\ Exactas y Tecnología Departamento de Botánica, Estafeta Universitaria, Panamá - Panamá \\ * Autor para correspondencia \\ tournefort@gmail.com
}

Submetido em $07 / 10 / 2013$

Aceito para publicação em 11/07/2014

\section{Resumo}

Clave para los géneros de Boraginaceae sensu lato nativos del Brasil. Este trabalho baseia-se na análise de espécimes de herbários, estudos de campo e revisão da literatura sobre os gêneros e espécies de Boraginaceae sensu lato representados no Brasil. É apresentada uma chave para os nove gêneros registrados, bem como comentários sobre a distribuição, hábitat e número das espécies brasileiras de cada gênero.

Palavras-chave: América do Sul; Boraginaceae; Brasil; Chave para gêneros

\section{Abstract}

This paper is based on the analysis of herbaria specimens, field studies, and literature review on sensu lato Boraginaceae genera and species represented in Brazil. A key to the nine genera registered is introduced, as well as comments on the distribution, habitat, and number of the Brazilian species from each genus.

Key words: Boraginaceae; Brazil; Key to genera; South America

\section{Introducción}

La familia Boraginaceae es cosmopolita y comprende 200 géneros y aproximadamente 2600 especies, distribuidas en las regiones templadas, subtropicales y tropicales del mundo (SMITH et al., 2004). En Brasil, ocurren 129 especies representadas en 9 géneros nativos los cuales ocurren en las diferentes formaciones vegetales del país (MELO et al., 2014).
La clasificación de Boraginaceae en subfamilias es basada fundamentalmente en el tipo de inserción al ovario, el patrón de ramificación de los estilos y en el tipo de fruto (AL-SHEHBAZ, 1991). Los géneros presentes en Brasil se asignan a las subfamilias: Cordioideae (Cordia, Varronia), Ehretioideae (Lepidocordia, Rotula), Heliotropioideae (Euploca, Heliotropium, Tournefortia) y Boraginoideae (Moritzia, Thaumatocaryon). 
La familia Boraginaceae comprende hierbas, subarbustos, arbustos, lianas o árboles de hojas simples, raro opuestas o verticiladas. Las inflorescencias son cimas, raro flores solitarias (como en Heliotropium lagoense (Warm.) Gürke); las flores son diclamídeas, pentámeras, con cinco estambres, inclusos o exsertos, ovario súpero y estilo terminal o ginobásico. El fruto es drupáceo (Cordia, Varronia), con uno o cuatro pirenos (Tournefortia) o esquizocárpico, con dos o cuatro núculas (Euploca, Heliotropium).

A pesar que en Brasil, la familia está representada en los diferentes biomas, poco se conoce sobre su taxonomía, aunque existan contribuciones importantes tales como: Fresenius (1857), Smith (1970), Guimarães et al. (1971), Taroda (1984), Taroda y Gibbs (1986a; 1986b; 1987), Stapf (2010) y Stapf y Silva (2013b). Recientemente, representantes de esta familia fueron referidos en listados de especies como: Dubs (1998), Harvey (1995), Munhoz y Proença (1998), Mendonça et al. (2000), como también en listados elaborados por Melo (2006), estos principalmente de las regiones del Nordeste y Centro-Oeste del país. Entre las floras locales y regionales podremos referir: Vitta (1992), Nagatani y Rossi (2000), Taroda y Silva (2002), Melo y França (2003), Zappi et al. (2003), Melo y Sales (2004; 2005), Melo (2007a), Melo y Andrade (2007) y Ranga et al. (2008). Recientemente, fueron publicadas nuevas especies de Boraginaceae del Brasil por Miller (2001), Melo y Semir (2006; 2009), Melo (2007b), Stapf et al. (2010) y Stapf y Silva (2013a). En el presente solo existe una clave que incluye los géneros nativos que ocurren en Brasil (MELO et al., 2007) basada en los cambios nomenclaturales y taxonómicos propuestos recién (BÖHLE; HILGER, 1997; DIANE et al., 2002; GOTTSCHLING; MILLER, 2006). Sin embargo, los géneros Cordia y Heliotropium sufrieron cambios nomenclaturales más recientemente (MILLER; GOTTSCHLING, 2007; MELO; SEMIR, 2009). Por este motivo, este trabajo presenta una clave actualizada para el reconocimiento de los géneros de Boraginaceae sensu lato, nativos del Brasil, con base a caracteres vegetativos y reproductivos.

\section{Material y Métodos}

Este trabajo fue basado en análisis de bibliografía especializada (FRESENIUS, 1857; JOHNSTON, 1927; SMITH, 1970; GUIMARÃES et al., 1971; TARODA, 1984; TARODA y GIBBS, 1986a; 1986b; GUIMARÃES; MAUTONE, 1989; MILLER; NOWICKE, 1990; CAVALHEIRO, 2004; MELO; SALES, 2004; 2005; GOTTSCHLING; MILLER, 2006; MELO; SEMIR, 2006; 2009; MELO, 2007a; 2007b; MELO; ANDRADE, 2007; MELO; LYRA-LEMOS, 2008; MELO et al., 2010; STAPF, 2010) y estudios de campo donde se observaron las poblaciones naturales y se colectaron muestras botánicas. También se analizaron especímenes, incluyendo colecciones históricas y tipos nomenclaturales, depositados en los siguientes herbarios brasileños y extranjeros (abreviaturas según HOLMGREN et al. (2007)): ALCB, B, BHCB, BM, CEN, CEPEC, CESJ, CH, COR, CPAP, EAC, ESA, GH, GUA, HAS, HBH, HBR, HRB, HRCB, HST, HTINS, HUEFS, HUFU, IAC, IAN, IBGE, ICN, INPA, IPA, JPB, K, M, MAC, MBM, MBML, MG, MHN, MO, MOSS, MUFAL, NY, P, PACA, PEL, PEUFR, R, RB, RFA, SJRP, SP, SPF, SPSF, TEPB, UB, UEC, UFMS, UFP, UFRN, VIES. El material examinado comprendió los diferentes géneros de Boraginaceae nativos del Brasil. La clave incluye caracteres vegetativos y reproductivos. El número de especies representado en los diferentes géneros fue basado en Melo et al. (2014). Adicionalmente, se presentan imágenes fotográficas del hábito, flores y/o frutos de representantes de los géneros que ocurren en el país.

\section{Resultados y Discusión}

Clave para los géneros de las Boraginaceae sensu lato nativos en Brasil

1. Estilo ginobásico; usualmente hierbas.

2. Inflorescencias sin brácteas; núculas sésiles 5. Moritzia

2. Inflorescencias con brácteas; núculas estipitadas......... 7. Thaumatocaryon

1. Estilo terminal; árboles o arbustos, rara vez hierbas.

3. Estilo dividido; estigmas 2 ó 4. 
4. Estigmas 2; semillas con endosperma.

4. Lepidocordia

4. Estigmas 4; semillas sin endosperma.

5. Arbustos con inflorescencias condensadas capituliformes ó espiciformes ó en cimas dicótomas; hojas usualmente serradas. 9. Varronia

5. Árboles o arbustos con inflorescencias en cimas abiertas o paniculifomes; hojas usualmente enteras.

1. Cordia

\section{Estilo entero; estigmas 1.}

6. Lóbulos de la corola rotáceos; fruto esquizocárpico. 6. Rotula

6. Lóbulos de la corola no rotáceos; fruto drupáceo o esquizocárpico.

7. Frutos drupáceos, presentando 2 ó 4 pirenos....

8. Tournefortia
7. Frutos esquizocárpicos, presentando 2 ó 4 mericarpos.

8. Anteras libres; fruto con 2 núculas

3. Heliotropium

8. Anteras generalmente coherentes; fruto con 4 núculas

2. Euploca

Antiphytum DC. ex Meisn. no está representado en Brasil y la especie Wigandia urens (Ruiz \& Pav.) Kunth, originalmente incluida en Hydrophyllaceae, no fue admitida en este trabajo lo cual incluye el concepto tradicional de la familia Boraginaceae. A continuación presentamos informaciones relacionadas al número de especies, la distribución geográfica y hábitat de los géneros presentes en el Brasil basándose en las especies nativas de Boraginaceae sensu lato.

1. Cordia L., Sp. P1. 1: 190-191. 1753. FIGURA 1: A-F

FIGURA 1: A-B. Cordia exaltata - A. Flores; B. Frutos inmaduros; C. Inflorescencia de C. glabrata; D. Inflorescencia de C. pilosa; E. Frutos maduros de C. rufescens; F. Inflorescencia de C. trachyphylla.
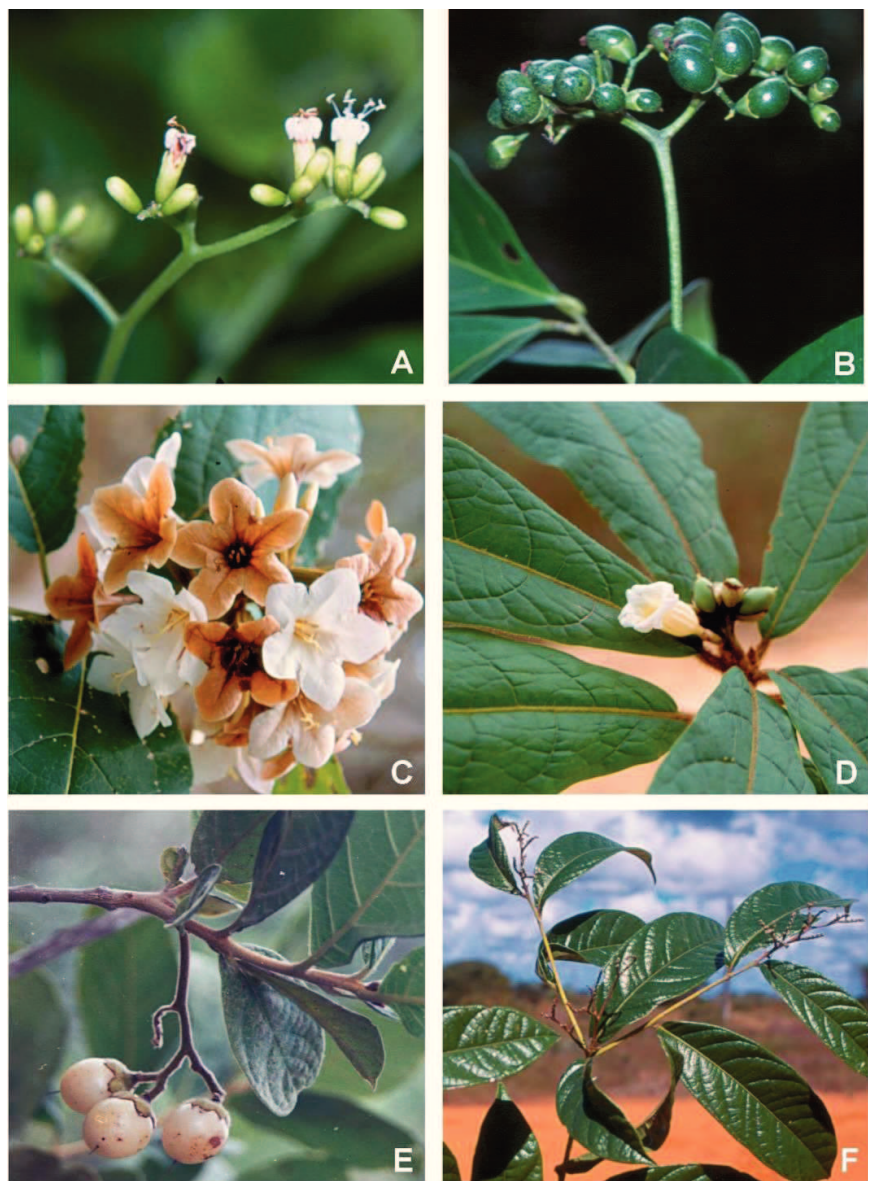
Comprende aproximadamente 250 especies, distribuidas en regiones tropicales y subtropicales del mundo. En Brasil, ocurren 50 especies de las cuales 26 son endémicas, generalmente crecen en la mata atlántica y en la región amazónica, algunas especies en caatingas y cerrados. El género fue recientemente reevaluado (GOTTSCHLING; MILLER, 2006) y actualmente incluye las especies de los géneros Auxemma Miers y Patagonula L. Por otro lado, las especies de una sección de Cordia (Varronia P. Browne) fueron elevadas al género Varronia (MILLER; GOTTSCHLING, 2007), que se caracteriza por su hábito arbustivo con tallos múltiples, márgenes de las hojas conspicuamente serrados y inflorescencias condensadas, espigadas, capitadas o cimas pequeñas.

2. Euploca Nutt., Trans. Amer. Philos. Soc., n.s., 5(6[3]): 189-190. 1837 [1836]. FIGURA 2: A-E
Género reciente segregado de Heliotropium, incluye las especies de Heliotropium sección Orthostachys Spach y las del género Schleidenia Endl. (DIANE et al., 2002), es reconocido principalmente por presentar frutos esquizocárpicos con 2 ó 4 núculas. Comprende cerca de 100 especies distribuidas en todo el mundo, siendo África, Australia y América tropical (especialmente América del Sur y México) los principales centros de diversidad. En Brasil hay 17 especies, de las cuales 6 son endémicas. Ocurren en todas las formaciones vegetales, especialmente en ambientes abiertos y secos (caatingas, cerrados y campos rupestres).

\section{Heliotropium L., Sp. Pl. 1: 130-131. 1753. FIGURAS} 2: F; 3: A-B

Este género fue recién segregado, incluyendo presentemente las especies que poseen anteras libres

FIGURA 2: A. Euploca polyphylla - inflorescencia; B. E. procumbens - inflorescencia; C-D. E. salicoides - C. Hábito; D. Inflorescencia; E. E. ternata - inflorescencia; F. Heliotropium curassavicum - hábito.
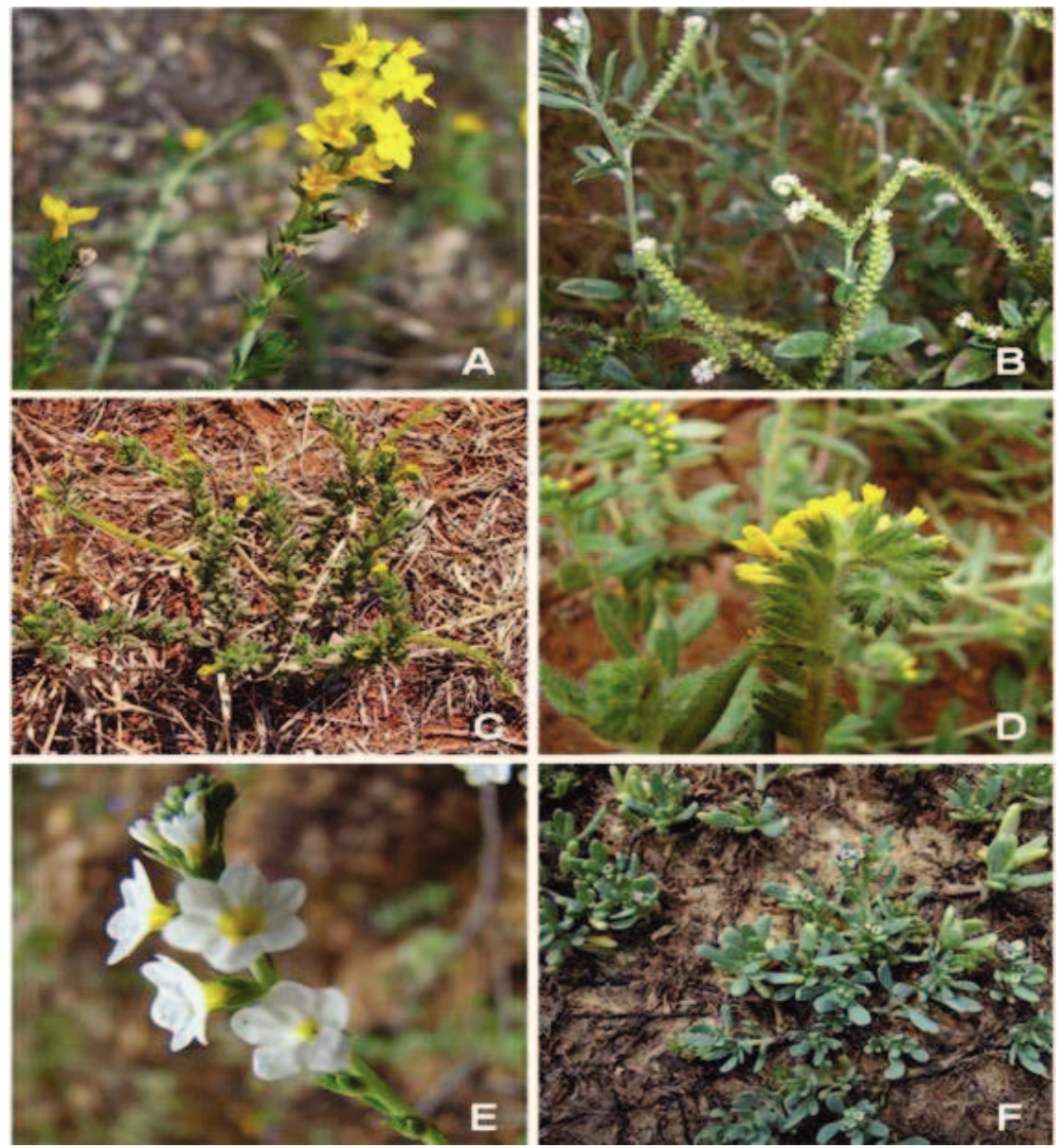
y frutos esquizocárpicos con 2 núculas (DIANE et al., 2002). Comprende cerca de 200 especies y los principales centros de diversidad son la región Turco-Iraní y América del Sur. En el Brasil fueron registradas nueve especies, distribuidas en todas las regiones del país, principalmente en la caatinga y en los "campos sulinos".

4. Lepidocordia Ducke, Arch. Jard. Bot. Rio de Janeiro 4: 170, t. 22. 1925.

Este género incluye especies con estilo dividido, presentando 2 estigmas y semillas con endosperma. Comprende dos especies neotropicales, Lepidocordia punctata Ducke y L. williamsii (I.M. Johnst.) J.S. Mill., distribuidas en América Central, Sur de México, y en el Noreste de América del Sur (Guiana, Suriname, Venezuela y Brasil). En Brasil sólo ocurre L. punctata, en los estados de Pará y Roraima, en la región amazónica.

Dibujos son presentados en Miller y Nowicke (1990).

5. Moritzia DC. ex Meisn., Pl. Vasc. Gen. 1: 280. 1840.

Comprende 3 especies distribuidas en América Central y América del Sur (Colombia, Venezuela y Brasil). En Brasil, ocurren dos especies endémicas, Moritzia dusenii I.M. Johnst. y M. ciliata (Cham.) DC. ex Meisn., en las regiones del Sureste y Sur del país.

Dibujos pueden ser encontrados en Smith (1970).

FIGURA 3: A. Inflorescencia de H. elongatum; B. H. indicum-hábito; C. Tournefortia paniculata-frutos; D. T. rubicunda-inflorescencia; E. T. salzmannii - hábito; F. T. volubilis - inflorescencia.
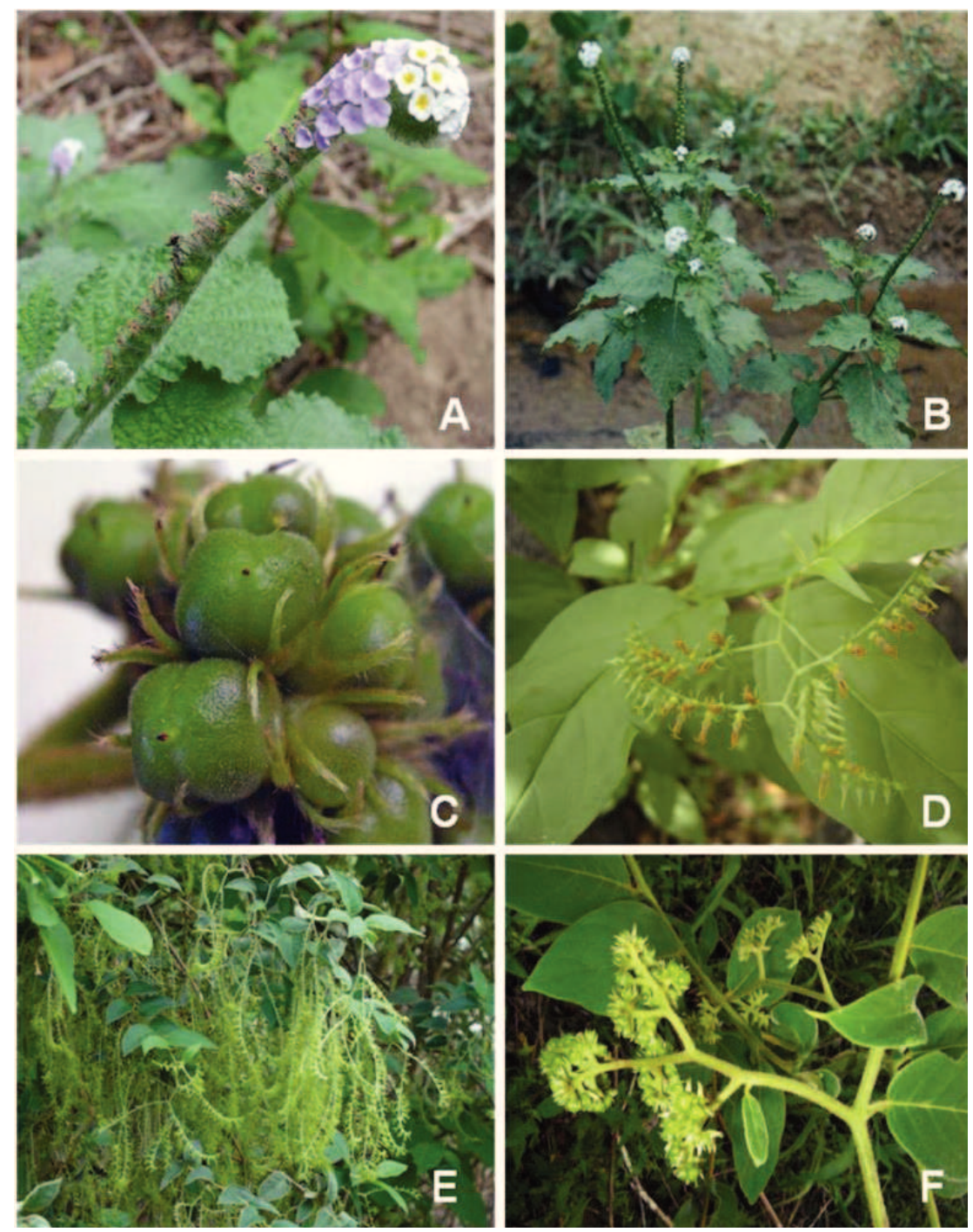
6. Rotula Lour., Fl. Cochinch. 1: 121. 1790.

Comprende 3 especies distribuidas en Brasil, África, Asia (India, Indochina y Sri-Lanka). En Brasil ocurren 2 especies endémicas del país, Rotula lycioides (Mart.) I. M. Johnst. y R. pohlii (Kuhlm.) E. F.Guim. \& Mautone. La primera está asociada a las regiones del Nordeste (Maranhão, Piauí, Ceará, Pernambuco y Bahia) y Sudeste (Minas Gerais y Rio de Janeiro) y la segunda, probablemente restringida al estado de Pará.

Dibujos pueden ser encontrados en Guimarães y Mautone (1989).

7. Thaumatocaryon Baill., Bull. Mens. Soc. Linn. Paris: 839. 1890.

Comprende 2 especies endémicas de América del Sur. En Brasil fueron registradas en Santa Catarina e Rio Grande do Sul (Sul): Thaumatocaryon dasyanthum (Cham.) I. M. Johnst. y T. tetraquetrum I. M. Johnst.
8. Tournefortia s.l. L., Sp. Pl. 1: 140-141. 1753. FIGURA 3: C-F

Incluye aproximadamente 130 especies, distribuidas principalmente en las regiones tropicales y subtropicales. Según Diane et al. (2002), Tournefortia no es un género monofilético y por esta razón las especies de Tournefortia sect. Cyphocyema I.M. Johnst. serán incorporadas al género Myriopus Small. La mayoría de las especies brasileñas de Tournefortia pertenecen a la sección Cyphocyema pero todavía no fueron propuestas todas las nuevas combinaciones en Myriopus. En este trabajo fue admitido el concepto tradicional del género, siendo registradas 18 especies en Brasil, de las cuales 5 son endémicas y ocurren principalmente en la región de la mata atlántica y amazónica (CAVALHEIRO et al., 2011; MELO et al., 2014).

9. Varronia P. Browne, Civ. Nat. Hist. Jamaica 172, pl. 13, f. 2. 1756. FIGURA 4: A-F

FIGURA 4: A-C. Varronia curassavica - A. Hábito; B. Inflorescencia; C. frutos maduros; D. Inflorescencia de V. leucocephala; E. Inflorescencia de V. multispicata; F. Inflorescencia de V. striata.
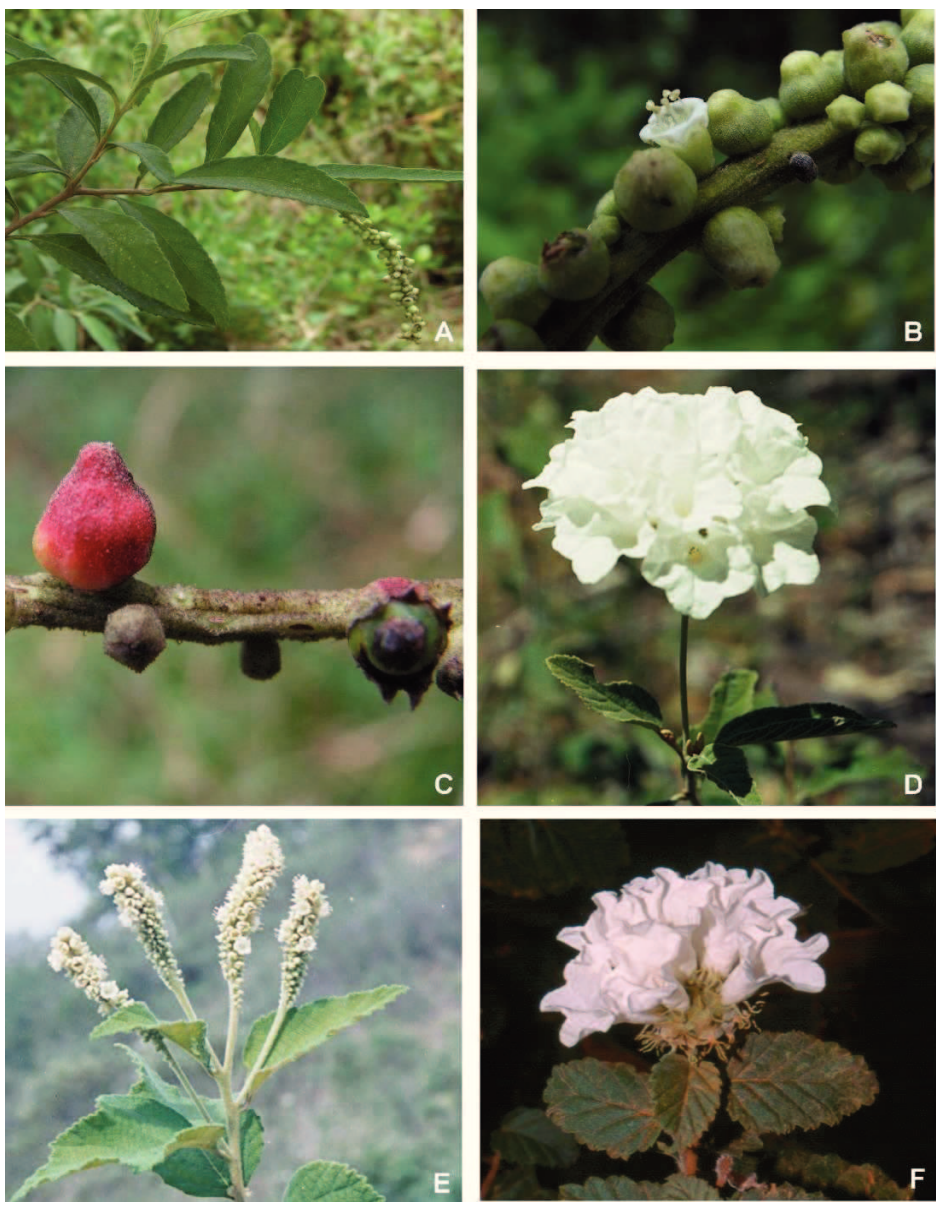
Comprende cerca de 100 especies neotropicales. En Brasil, ocurren 28 especies, siendo 13 especies endémicas. Este género ocurre principalmente en los biomas caatinga y cerrado.

\section{Agradecimientos}

A los herbarios brasileños y extranjeros listados, por el envío de las colecciones ó el permiso para consulta de las mismas durante las visitas hechas por los autores. J.I.M. Melo agradece al CNPq (Consejo Nacional de Desarrollo Científico y Tecnológico) por la concesión de la Beca de Productividad en Investigación.

\section{Referencias}

AL-SHEHBAZ, I. A. The genera of Boraginaceae in the Southeastern United States. Journal of the Arnold Arboretum, Cambridge, v. 1, p. 1-169, 1991.

BÖHLE, U. R.; HILGER, H. H. Chloroplast DNA systematics of "Boraginaceae" and related families: a goodbye to the old familiar concept of 5 subfamilies. Scripta Botanica Belgica, Bruxèlles, v. 15, p. 30, 1997.

CAVALHEIRO, L. Estudos taxonômicos das espécies brasileiras extra-amazônicas de Tournefortia L. (Boraginaceae s.l.). 2004. 119 f. Dissertação (Mestrado em Botânica) - Universidade Estadual Paulista Júlio de Mesquita Filho, Rio Claro. 2004.

CAVALHEIRO, L.; RANGA, N. T.; FURLAN, A. Tournefortia L. (Boraginaceae): espécies do Brasil extra-amazônico. Hoehnea, São Paulo, v. 38, p. 221-242, 2011.

DIANE, N.; FÖRTHER, H.; HILGER, H. H. A systematic analysis of Heliotropium, Tournefortia, and allied taxa of the Heliotropiaceae (Boraginales) based on ITS1 sequences and morphological data. American Journal of Botany, Saint Louis, v. 89, p. 287-295, 2002. DUBS, B. Boraginaceae. In: DUBS, B. (Ed.). Prodromus Florae Matogrossensis. The botany of Mato Grosso. Vol. 3. Küsnacht: Bertrona-Verlag, Series B, 1998. p. 40-41.

FRESENIUS, G. Cordiaceae, Heliotropieae et Borragineae. In: MARTIUS, C. F. P. (Ed.). Flora brasiliensis. Vol. 9. München: Flora Brasiliensis, 1857. p. 1-60.

GOTTSCHLING, M.; MILLER, J. S. Clarification of the taxonomic position of Auxemma, Patagonula and Saccelium (Cordiaceae, Boraginales). Systematic Botany, Saint Louis, v. 31, p. 361-367, 2006.

GUIMARÃES, E. F.; BARROSO, G. M.; ICHASO, C. L. F.; BASTOS, A. R. Flora da Guanabara: Boraginaceae. Rodriguésia, Rio de Janeiro, v. 38, p. 194-220, 1971.

GUIMARÃES, E. F.; MAUTONE, L. O gênero Rotula Lour. (Boraginaceae). Revista Brasileira de Biologia, São Carlos, v. 49, p. 61-74, 1989.

HARVEY, Y. B. Boraginaceae. In: STANNARD, B. L. (Ed.). Flora of the Pico das Almas, Chapada Diamantina - Bahia, Brasil. Kew: Royal Botanic Gardens, 1995. p. 155-157.
HOLMGREN, P. K.; HOLMGREN, N. H.; BARNETT, L. C. Index Herbariorum. Part I. The Herbaria of the world. 2007. Disponível em: <http: //sci.web.nybg.org/science2/IndexHerbariorum.as.>. Acceso en: 20 mar. 2013.

JOHNSTON, I. M. Studies in the Boraginaceae VI: A revision of the South American Boraginoideae. Contributions from the Gray Herbarium of Harvard University, Cambridge, v. 78, p. 3-118, 1927.

MELO, E.; FRANÇA, F. Flora de Grão Mogol, Minas Gerais: Boraginaceae. Boletim de Botânica da Universidade de São Paulo, São Paulo, v. 21, p. 127-129, 2003.

MELO, J. I. M. Boraginaceae. In: BARBOSA, M. R. V.; SOTHERS, C.; MAYO, S. J.; GAMARRA-ROJAS, C. F. L.; MESQUITA, A.C. (Ed.). Checklist das plantas do nordeste brasileiro: Angiospermas e Gimnospermas. Brasília: Ministério da Ciência e Tecnologia, 2006. 49 p.

MELO, J. I. M. Taxonomia e distribuição dos gêneros Euploca Nutt. e Heliotropium L. (Heliotropiaceae) para o Brasil. 2007a. 154 f. Tese (Doutorado em Botânica) - Universidade Federal Rural de Pernambuco, Recife. 2007a.

MELO, J. I. M. Uma nova espécie de Tournefortia L. (Boraginaceae s.l.) para o Nordeste do Brasil. Hoehnea, São Paulo, v. 34, p. $155-$ $158,2007 \mathrm{~b}$.

MELO, J. I. M.; ANDRADE, W. M. Boraginaceae s.l. A. Juss. em uma área de Caatinga da ESEC Raso da Catarina, Bahia - Brasil. Acta Botanica Brasilica, São Paulo, v. 21, p. 369-378, 2007.

MELO, J. I. M.; LYRA-LEMOS, R. P. Sinopse taxonômica de Boraginaceae s.l. A. Juss. no estado de Alagoas - Brasil. Acta Botanica Brasilica, São Paulo, v. 22, p. 701-710, 2008.

MELO, J. I. M.; SALES, M. F. Heliotropium L. (Boraginaceae Heliotropioideae) de Pernambuco, Nordeste do Brasil. Rodriguésia, Rio de Janeiro, v. 55, p. 65-87, 2004.

MELO, J. I. M.; SALES, M. F. Boraginaceae A. Juss. na região de Xingó: Alagoas e Sergipe. Hoehnea, São Paulo, v. 32, p. 369-380, 2005.

MELO, J. I. M.; SEMIR, J. Euploca rodaliae J.I.M. Melo \& Semir - A new species of Euploca (Heliotropiaceae) from Brazil. Candollea, Gèneve, v. 61, p. 453-456, 2006.

MELO, J. I. M.; SEMIR, J. Two new Brazilian species and new combinations in Euploca (Heliotropiaceae). Kew Bulletin, Kew, v. 64, p. 285-289, 2009.

MELO, J. I. M.; SILVA, L. C.; STAPF, M. N. S.; RANGA, N. T. Boraginaceae. In: FORZZA, R. C. et al. (Ed.). Catálogo de plantas e fungos do Brasil. Rio de Janeiro: Jardim Botânico do Rio de Janeiro, 2010. p. 773-777.

MELO, J. I. M.; SILVA, L. C.; STAPF, M. N. S.; RANGA, N. T. Boraginaceae. In: FORZZA, R. C. et al. (Ed.). Lista de espécies da flora do Brasil. 2014. Rio de Janeiro: Jardim Botânico do Rio de Janeiro. Disponível em: <http: //floradobrasil.jbrj.gov.br/2010/ FB016555>. Acceso en: 12 mayo 2014.

MELO, J. I. M.; PAULINO, R. C.; SILVA, F. V. Chave ilustrada para os gêneros de Boraginaceae sensu lato nativos do Brasil. Revista Caatinga, Mossoró, v. 20, p. 172-180, 2007.

MENDONÇA, R. C.; FELFILI, J. M.; FAGG, C. W.; SILVA, M. A.; FILGUEIRAS, T. S.; WALTER, B. M. T. Florística da região do Espigão Mestre do São Francisco, Bahia e Minas Gerais. Boletim 
do Herbário Ezechias Paulo Heringer, Brasília, v. 6, p. 38-94, 2000.

MILLER, J. S. New Boraginaceae from Tropical America: 4. Three new species of Cordia from South America. Novon, Saint Louis, v. 11, p. 421-428, 2001.

MILLER, J. S.; GOTTSCHLING, M. Generic classification in the Cordiaceae (Boraginales): Resurrection of the genus Varronia P. Br. (Cordiaceae). Taxon, Viena, v. 56, p. 163-169, 2007.

MILLER, J. S.; NOWICKE, J. W. Dioecy and a reevaluation of Lepidocordia and Antrophora (Boraginaceae: Ehretioideae). American Journal of Botany, Saint Louis, v. 77, p. 543-551, 1990.

MUNHOZ, C. B. R.; PROENÇA, C. E. B. Composição florística do município de Alto Paraíso de Goiás na Chapada dos Veadeiros. Boletim do Herbário Ezechias Paulo Heringer, Brasília, v. 3, p. 102-150, 1998.

NAGATANI, Y.; ROSSI, L. Flora fanerogâmica da Reserva do Parque Estadual das Fontes do Ipiranga - São Paulo, Brasil: 141 Boraginaceae. Hoehnea, São Paulo, v. 27, p. 95-98, 2000.

RANGA, N. T.; MELO, J. I. M. Boraginaceae. In: DALY, D. C.; SILVEIRA, M. (Ed.). Primeiro Catálogo da Flora do Acre, Brasil. Rio Branco/New York: Universidade Federal do Acre/The New York Botanical Garden, 2008. p. 154-155.

SMITH, L. B. Boragináceas. In: REITZ, P. R. (Ed.). Flora Ilustrada Catarinense. Itajaí: Herbário Barbosa Rodrigues, 1970. 85 p.

SMITH, N.; MORI, S. A.; HENDERSON, A.; STEVENSON, D. W.; HEALD, S. Flowering plants of the Neotropics. New York: The New York Botanical Garden, Princeton University Press, 2004. $593 \mathrm{p}$.

STAPF, M. N. S. Nomenclatural notes on Varronia (Boraginaceae s.l.) in Brazil. Rodriguésia, Rio de Janeiro, v. 61, p. 133-135, 2010.

STAPF, M. N. S.; SILVA, T. R. S. Typifications in Cordia (Cordiaceae), with an assessment of the status of Cordia blanchetii. Kew Bulletin, Kew, v. 68, p. 355-359, 2013a.
STAPF, M. N. S.; SILVA, T. R. S. Four new species of genus Cordia (Cordiaceae, Boraginales) from Brazil. Brittonia, New York, v. 65, p. 191-199, 2013b.

STAPF, M. N. S.; RANGA, N. T.; SILVA, T. R. A new species of Cordia (Cordiaceae, Boraginales) from Brazil. Novon, Saint Louis, v. 20, p. 212-214, 2010.

TARODA, N. Taxonomic studies on Brazilian species of Cordia (Boraginaceae). 1984. $231 \mathrm{f}$. Thesis (PhD in Botany) - University of Saint Andrews, Saint Andrews. 1984.

TARODA, N.; GIBBS, P. E. Studies on the genus Cordia L. (Boraginaceae) in Brazil. 1. A new infrageneric classification and conspectus. Revista Brasileira de Botânica, São Paulo, v. 9, p. 31-42, 1986a.

TARODA, N.; GIBBS, P. E. A revision of the Brazilian species of Cordia subgenus Varronia (Boraginaceae). Notes from the Royal Botanical Garden of Edinburgh, Edinburgh, v. 44, p. 105-140, 1986b.

TARODA, N.; GIBBS, P. E. Studies on the genus Cordia L. (Boraginaceae) in Brazil. 2. An outline taxonomic revision of subgenus Myxa Taroda. Hoehnea, São Paulo, v. 14, p. 31-52, 1987.

TARODA, N.; SILVA, L. C. Boraginaceae. In: MELO, M. M. R. F.; BARROS, F.; WANDERLEY, M. G. L.; KIRIZAWA, M.; JUNG-MENDAÇOLLI, S. L.; CHIEA, S. A. C. (Ed.). Flora fanerogâmica da Ilha do Cardoso (São Paulo, Brasil). Vol. 9. São Paulo: Instituto de Botânica de São Paulo, 2002. p. 105-114.

VITTA, F. A. Flora da Serra do Cipó, Minas Gerais: Boraginaceae. Boletim de Botânica da Universidade de São Paulo, São Paulo, v. 13, p. 235-239, 1992.

ZAPPI, D. C.; LUCAS, E.; STANNARD, B. L.; LUGHADA, E. N.; PIRANI, J. R.; QUEIROZ, L. P.; ATKINS, S.; HIND, D. J. N.; GIULIETTI, A. M.; HARLEY, R. M.; CARVALHO, A. M. Lista das plantas vasculares de Catolés, Chapada Diamantina, Bahia, Brasil. Boletim de Botânica da Universidade de São Paulo, São Paulo, v. 21, p. 345-39, 2003. 